\title{
Input-Driven Oscillations in Networks with Excitatory and Inhibitory Neurons with Dynamic Synapses
}

\author{
Daniele Marinazzo \\ Daniele.Marinazzo@ba.infn.it \\ Department of Biophysics, Radboud University of Nijmegen, 6525 EZ Nijmegen, \\ The Netherlands; TIRES - Center of Innovative Technologies for Signal Detection and \\ Processing and Dipartimento Interateneo di Fisica, Università di Bari, 70125, Bari, \\ Italy; and Istituto Nazionale di Fisica Nucleare, Sezione di Bari, 70125 Bari, Italy
}

\author{
Hilbert J. Kappen \\ B.Kappen@science.ru.nl \\ Stan C. A. M. Gielen \\ S.Gielen@science.ru.nl \\ Department of Physics, Radboud University of Nijmegen, 6525 EZ Nijmegen, \\ The Netherlands
}

Previous work has shown that networks of neurons with two coupled layers of excitatory and inhibitory neurons can reveal oscillatory activity. For example, Börgers and Kopell (2003) have shown that oscillations occur when the excitatory neurons receive a sufficiently large input. A constant drive to the excitatory neurons is sufficient for oscillatory activity. Other studies (Doiron, Chacron, Maler, Longtin, \& Bastian, 2003; Doiron, Lindner, Longtin, Maler, \& Bastian, 2004) have shown that networks of neurons with two coupled layers of excitatory and inhibitory neurons reveal oscillatory activity only if the excitatory neurons receive correlated input, regardless of the amount of excitatory input. In this study, we show that these apparently contradictory results can be explained by the behavior of a single model operating in different regimes of parameter space. Moreover, we show that adding dynamic synapses in the inhibitory feedback loop provides a robust network behavior over a broad range of stimulus intensities, contrary to that of previous models. A remarkable property of the introduction of dynamic synapses is that the activity of the network reveals synchronized oscillatory components in the case of correlated input, but also reflects the temporal behavior of the input signal to the excitatory neurons. This allows the network to encode both the temporal characteristics of the input and the presence of spatial correlations in the input simultaneously. 


\section{Introduction}

The central nervous system can display a wide spectrum of spatially synchronized, rhythmic oscillatory patterns of activity with frequencies in the range from $0.5 \mathrm{~Hz}$ ( $\delta$ rhythm), $20 \mathrm{~Hz}$ ( $\beta$ rhythm), to 40 to $80 \mathrm{~Hz}$ ( $\gamma$ rhythm) and even higher up to $200 \mathrm{~Hz}$ (for a review, see Gray, 1994). In the past two decades, evidence has been presented that synchronized activity and temporal correlation are fundamental tools for encoding and exchanging information for neuronal information processing in the brain (Singer \& Gray, 1995; Singer, 1999; Reynolds \& Desimone, 1999). In particular, it has been suggested that clusters of cells organize spontaneously into flexible groups of neurons with similar firing rates, but with a different temporal correlation structure. However, despite the fact that synchronization of groups of neurons has been the subject of intense research efforts in many studies, the functional role of synchronized activity is still a topic of debate (Gray, 1994; Fries, 2005). A longstanding, fundamental question is when synchrony can emerge and how cell properties, synaptic interactions, and network architecture interact to determine the nature of synchronous states in a large neural network. Most theoretical studies have investigated synchronization of neuronal activity in fully connected or sparsely connected networks under assumptions of weak or strong coupling (see, e.g., Ariaratnam \& Strogatz, 2001; Mirollo \& Strogatz, 1990; Ernst, Pawelzik, \& Geisel, 1995; Hansel \& Mato, 1993; Hansel, Mato, \& Meunier, 1995; van Vreeswijk, 2000).

Several experimental studies have shown that the grouping of neurons with synchronized firing depends on stimulus properties and on instruction to the subject or on attention (Reynolds, Chelazzi, \& Desimone, 1999; Schoffelen, Oostenveld, \& Fries, 2005). Therefore, both network properties, stimulus-driven feedforward (bottom-up) and feedback (top-down) mechanisms, are involved and should be incorporated in an explanatory model. These concepts are compatible with neurophysiological studies, which have suggested that synchronous oscillatory activity may be entrained by synchronous rhythmic inhibition originating from fast-spiking inhibitory interneurons (Buzsáki, Leung, \& Vanderwolf, 1983; Lytton \& Sejnowski, 1991), which has been an important reason to study networks with excitatory and inhibitory neurons.

Based on this insight, two different models have been proposed in the literature to explain the emergence of synchronized neuronal activity. Doiron, Lindner, Longtin, Maler, \& Bastian (2004) presented a network with a layer of excitatory neurons with stochastic input and delayed inhibitory feedback of the summed activity of the excitatory neurons to the excitatory neurons. This model revealed the remarkable property that oscillations were observed in the excitatory neurons in response to spatially correlated stimuli but not to uncorrelated input. It was the spatial correlation in stimuli, not the total power of the stimulus, that was essential for the network to display oscillatory activity. The frequency of oscillation was determined by 
the effective delay in the feedback loop and was not related to the temporal properties of spatially correlated input.

Although the architecture with interacting populations of excitatory and inhibitory neurons in the model by (Doiron, Chacron, Maler, Longtin, \& Bastian, 2003; Doiron et al., 2004) was similar to the architecture proposed by Börgers and Kopell (2003), the behavior of the latter model, which was proposed to explain oscillations in the gamma range $(25-80 \mathrm{~Hz})$, was different from that proposed by Doiron et al. The Börgers and Kopell model consists of two interacting layers of excitatory and inhibitory neurons without a pure time delay in the feedback loop. This model assumes a considerably longer time constant for the decay of inhibition than for the decay of excitation. It reveals synchronous rhythmic spiking due to a constant excitatory drive to the excitatory neurons. The excitatory neurons provide diverging input to the inhibitory cells, which inhibit (by divergent feedback to the excitatory neurons, where each inhibitory cell projects to multiple excitatory cells) and thereby synchronize the excitatory cells. With the proper parameters, this network starts to oscillate as soon as the power of the external input to the excitatory neurons is sufficiently large, regardless of spatial correlations in the input. This result may seem at odds with the results by Doiron et al. (2003), since Doiron et al. describe that oscillations occur only for spatially correlated input, regardless of the intensity of the external input. In our study, we present a more general model and demonstrate that the models by Doiron et al. $(2003,2004)$ and by Börgers and Kopell (2003) are a special case of a more general model, which reveals a qualitatively different behavior in different regimes of parameter space.

In order to understand the reasons for the apparent discrepancy, we used the same architecture as Doiron et al. and Börgers and Kopell, with a layer of excitatory neurons and a layer of inhibitory neurons. External input is provided to the excitatory neurons, which feed their output to the inhibitory neurons. In addition to the external input, the excitatory neurons receive feedback from the inhibitory neurons. Regarding the type of neuron, Börgers and Koppell used the theta model (Ermentrout \& Kopell, 1986) whereas Doiron et al. $(2003,2004)$ used the leaky integrate-and-fire (LIF) model. The LIF model is an approximation of the more physiological Hodgkin-Huxley model, but the standard Hodgkin-Huxley neuron also can well be approximated by the theta model (see, e.g., Ermentrout \& Kopell, 1986; Hoppensteadt \& Izhikevich, 1997; Gutkin \& Ermentrout, 1998). In this study we choose the LIF neuron, because Lindner et al. (Lindner \& Schimansky-Geier, 2001; Lindner, Schimansky-Geier, \& Longtin, 2002; Lindner, Doiron, \& Longtin, 2005) provided a method for an analytical analysis as an approximation for the dynamics of the LIF neuron. This allowed us to derive analytical expressions, which could be compared with the results of numerical analyses to explain the oscillatory behavior of the network. We also did some simulations using the standard Hodgkin-Huxley neuron, which gave the same results. 
In this study we add dynamic synapses (Tsodyks \& Markram, 1997) to the model. Experimental studies have shown that the efficacy with which a synapse can transmit an action potential depends on the recent history of the synapse itself. The model by Tsodyks, Pawelzik, and Markram (1998) for dynamic synapses incorporates the property that presynaptic activity gives rise to depletion of neurotransmitter. This makes the synapse less effective when the firing rate of presynaptic activity arriving at the synapse increases. This dynamical behavior, called short-term depression, has been explained and modeled in detail (Tsodyks et al., 1998). Tsodyks, Uziel, and Markram (2000) and Pantic, Torres, and Kappen (2003) have explained how temporal coding, synchronization, and coincidence detection are greatly affected by a time-varying synaptic strength. Based on the results in the literature, we hypothesized that incorporating dynamic synapses in the network should made the network behavior robust for a relatively large range of input characteristics. Incorporating dynamic synapses also gave another surprising result. The model by Doiron et al. (2004) produced a resonance when the input was spatially correlated only when the feedback gain was relatively small. With dynamic synapses, this behavior became robust for a large range of feedback gains. Moreover, the model with dynamic synapses both shows a resonance peak to indicate spatial correlation at the input and preserves the temporal characteristics of the spatially correlated input. This is a novel finding, which is important for modeling sensory (e.g., visual) information processing in combination with rhythmic activity (such as $\theta-, \beta-$, and $\gamma$-rhythms) in cortex.

\section{Model Description}

The basic architecture of our model is very similar to that of Doiron et al. (2004) and is described in Figure 1: a population of leaky integrate-andfire (LIF) neurons labeled $1,2, \ldots, N_{E}$, with external inputs $s_{1}, \ldots, s_{N_{E}}$. In this study, $N_{E}$ was equal to 100 . The output of these neurons provides excitatory input to another LIF neuron, which provides inhibitory feedback with gain $g$ to the excitatory neurons. In some of our simulations, the single inhibitory LIF neuron was replaced by a set of 20 LIF neurons, which project by inhibitory synapses with strength $\mathrm{g} / 20$ to each of the excitatory neurons. One of differences between our model and that by Doiron et al. $(2003,2004)$ is that the inhibitory feedback in the model by Doiron et al. (2004) was obtained by convolution of a linear summation of action potentials of the excitatory neurons, whereas we used the spike output of an LIF neuron (or of 20 LIF neurons) as inhibitory feedback to the excitatory neurons. As we will show later (see Figures 2 and 3), this affects only to a minor extent the responses of the model, as long as the membrane time constant of the leaky integrate-and-fire neuron is relatively long. 


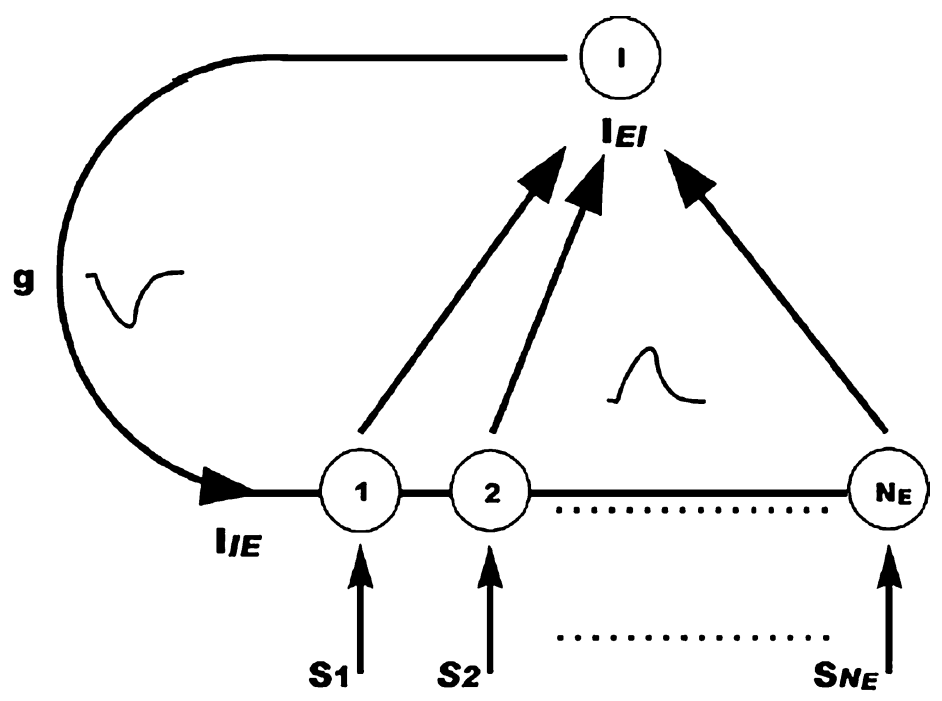

Figure 1: Model architecture describing the external input $s_{i}$ to the excitatory neurons. The output of the excitatory neurons projects to the inhibitory neuron with an effective input current $I_{E I}$. The output of the inhibitory neuron is fed back to the excitatory neurons by the inhibitory current $I_{I E}$.

The dynamics of the membrane potential V of the LIF neurons satisfies

$$
\frac{d V(t)}{d t}=-\left(V(t)-V_{r}\right)+I(t)
$$

where time is measured in units of the membrane time constant $\tau_{m}$ and the membrane resistance is normalized to one. Every time the potential of the $j$ th neuron reaches the threshold value $V_{t h}$, a spike is fired. This resets the potential to the rest potential $V_{r}$ and remains bound to this value for an absolute refractory period $\tau_{\text {Ref }}$. As in Doiron et al. $(2003,2004)$, we have set $V_{r}=0, V_{t h}=1$, and $\tau_{\text {Ref }}=3 \mathrm{~ms}$.

Each excitatory neuron $j\left(j \in\left\{1, \ldots, N_{E}\right\}\right)$ receives an input $I_{j}(t)$,

$$
I_{j}(t)=\mu+\eta_{j}(t)+\sigma\left\lfloor\sqrt{1-c} \xi_{j}(t)+\sqrt{c} \xi_{G}(t)\right\rfloor
$$

which consists of a constant base current $\mu$, internal gaussian white noise $\eta_{j}(t)$ with intensity $D$, and a stimulus $s_{j}(t)=\sigma\left(\sqrt{1-c} \xi_{j}(t)+\sqrt{c} \xi_{G}(t)\right)$ where $\xi_{j}(t)$ and $\xi_{G}(t)$ are both gaussian white noise with zero mean and unit power. Varying $c$ increases or decreases the degree of spatial correlation of the external stimuli, while the total input power to each neuron remains constant. In addition to this input, the excitatory neurons receive 
inhibitory feedback (see Figure 1), which will be defined in equation 2.4 and the text following that equation.

The series of spikes $s_{j}^{E}(t)$ of neuron $j$ in this layer of input neurons provides excitatory input to the inhibitory LIF neuron, which has a constant base current $\mu$. The input current $I_{E I}$ to the inhibitory neurons due to input from the excitatory neurons is given by the convolution of the sum of the spike trains of the excitatory neurons and a standard $\alpha$-function:

$$
I_{E I}(t)=K_{\tau}^{E} * S T_{E}(t)=\int_{0}^{\infty} S T_{E}(t-\tau) \alpha^{2} \tau e^{-\alpha \tau} d \tau,
$$

where $S T_{E}(t)=\sum_{j=1}^{N_{E}} s_{j}^{E}(t)$ is the sum of the spike trains of all excitatory neurons.

The base current to the neurons is too small to reach threshold. Therefore, neurons fire only in response to excitatory spike input.

Just like the excitatory neurons, the inhibitory LIF neuron has a constant base current $\mu$ and internal noise $\eta(t)$ with the same variance as that for the excitatory neurons. In addition, it receives the input $I_{E I}(t)$, as defined by equation 2.3. The action potentials $s^{I}(t)$ of the inhibitory neuron provide an inhibitory synaptic current to the excitatory neurons after a time delay $\tau_{D}$. The shape of the postsynaptic potential in the excitatory neurons due to input from the inhibitory neuron will be represented in further equations by $K_{\tau}^{I}$, which will be defined after the introduction of equation 2.4 .

For the case of dynamic synapses, their effective strength is governed by three parameters obeying the following equations (Tsodyks \& Markram, 1997),

$$
\begin{aligned}
& \frac{d x}{d t}=z / \tau_{r e c}-U x s^{I}\left(t-\tau_{D}\right) \\
& \frac{d y}{d t}=-y / \tau_{\text {in }}+U x s^{I}\left(t-\tau_{D}\right) \\
& \frac{d z}{d t}=y / \tau_{\text {in }}-z / \tau_{\text {rec }}
\end{aligned}
$$

where $x, y$, and $z$ are the fraction of synaptic resources in the recovered, active, and inactive state, respectively. Without any spike input, all neurotransmitter is recovered, and the fraction of available neurotransmitter is one: $x(t)=1$. After each spike arriving at the synapse, a fraction $U$ of the available (recovered) neurotransmitter is released. Note that the spike input from the inhibitory neuron $s^{I}(t)$ is delayed by $\tau_{D}$. The fraction $y$ of active neurotransmitter is then inactivated into the inactive state $z$. $\tau_{\text {in }}$ is the time constant of the inactivation process, and $\tau_{\text {rec }}$ is the recovery time constant for conversion of the inactive to the active state. With dynamic synapses, 
the total postsynaptic current $I_{I E}(t)$ is proportional to the fraction of neurotransmitter in the active state, that is, $I_{I E}(t)=g y(t)(g<0$ because of the inhibitory feedback). The total input to the excitatory neuron $j$ is defined by $I_{j}(t)+I_{I E}(t)$, where $I_{j}(t)$ is defined in equation 2.2.

With this model, we can easily change to a situation with static synapses by setting $x(t)=1$ for all $t$ (or, equivalently, $\tau_{\text {rec }} \rightarrow 0$ ). Note that equation 2.4 includes an exponential decay for the postsynaptic potential with a time constant $\tau_{\text {in }}$ in response to each presynaptic action potential. This is true for the case of both dynamic and static synapses.

We used the following parameter values in the model simulations: $N_{E}=$ $100 ; \tau_{m}=6 \mathrm{~ms}$ for all neurons except when explicitly mentioned otherwise; base current $\mu=0.5$; intensity of the internal gaussian white noise $D=$ $0.08 ; \sigma=0.4 ; \alpha=18 \mathrm{~ms}^{-1} ; \tau_{i n}=3 \mathrm{~ms} ; \tau_{r e c}=800 \mathrm{~ms}$; and time delay in the feedback loop $\tau_{D}=6 \mathrm{~ms}$. All simulations were integrated using a Euler integration scheme with a time step of $0.05 \mathrm{~ms}$.

Along similar lines as outlined in Doiron et al. (2004; see the appendix), we obtain the following expression for the spike train power spectral density of the excitatory neurons when feedback is provided by an LIF neuron

$$
\begin{aligned}
& \left\langle s_{j}^{E}(\omega) s_{j}^{*^{E}}(\omega)\right\rangle=\left\langle s_{0, j}^{E}(\omega) s_{0, j}^{*^{E}}(\omega)\right\rangle+\sigma^{2}\left|A^{E}(\omega)\right|^{2}+\ldots+c \sigma^{2}\left|A^{E}(\omega)\right|^{2} \\
& \times \frac{2 \Re\left(A^{E}(\omega) g e^{-i \omega \tau_{D}} K_{\tau}^{I}(\omega) A^{I}(\omega) K_{\tau}^{E}(\omega)\right)-\left|A^{E}(\omega) g K_{\tau}^{I}(\omega) A^{I}(\omega) K_{\tau}^{E}(\omega)\right|^{2}}{\left|1-A^{E}(\omega) g e^{-i \omega \tau_{D}} K_{\tau}^{I}(\omega) A^{I}(\omega) K_{\tau}^{E}(\omega)\right|^{2}}
\end{aligned}
$$

Here, $s_{j}^{E}(\omega)$ and $s_{0, j}^{E}(\omega)$ represent the spike train in the presence and absence, respectively, of the external stimulus and feedback. $K_{\tau}^{E}(\omega)$ and $K_{\tau}^{I}(\omega)$ represent the postsynaptic dynamics as defined in equations 2.3 and 2.4, respectively, in the frequency domain, and $A^{E}(\omega)$ and $A^{I}(\omega)$ represent the susceptibility in the frequency domain with respect to the input of the excitatory neurons and the inhibitory neurons, respectively (for details see Doiron et al., 2004; Lindner \& Schimansky-Geier, 2001; Lindner et al., 2002). $\mathfrak{R}(C)$ represents the real-valued part of the complex number $C$. This result was obtained with the assumptions that $\left\langle s_{0, j}^{E}(\omega) s_{k}^{*^{E}}(\omega)\right\rangle=\left\langle s_{0, j}^{E}(\omega) I_{j}^{*}(\omega)\right\rangle=$ $\left\langle s_{j}^{E}(\omega) \xi_{k}^{*}(\omega)\right\rangle=0$ for $j \neq k$ and for $N \rightarrow \infty$ so as to neglect terms of order $1 / N$ and higher. A derivation of equation 2.5 is given in the appendix. Resonance is obtained when the denominator of the last term in equation 2.5 is minimal, which depends mainly on the effective time delay (i.e., the sum of the pure time delay $\tau_{D}$, the frequency-dependent phase shifts due to the dynamics $A^{E}(\omega)$ and $A^{I}(\omega)$ of the LIF neurons, and the synaptic functions $K_{\tau}^{E}(\omega)$ and $\left.K_{\tau}^{I}(\omega)\right)$ in the feedback loop. 


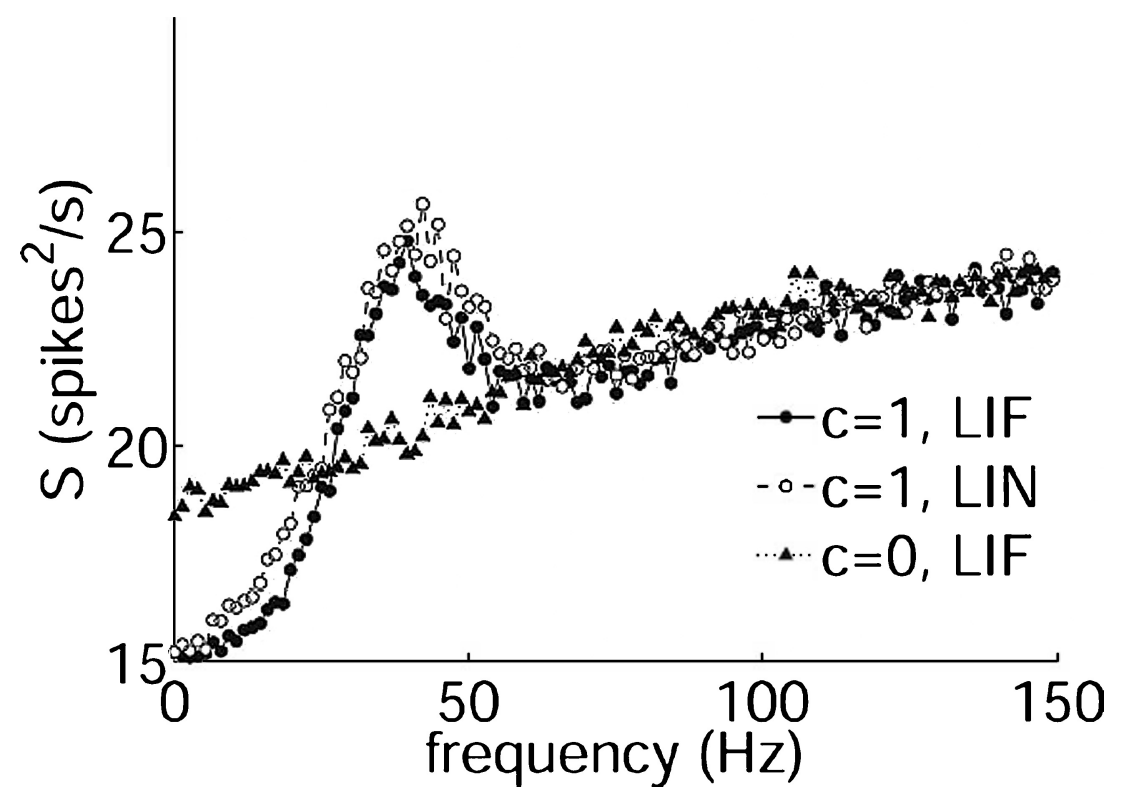

Figure 2: Spike train power spectra (defined by equation 2.5) of an excitatory neuron for spatially correlated $(c=1)$ and uncorrelated $(c=0)$ input when inhibition is provided by an LIF neuron (filled symbols) and for correlated input when inhibition is provided by a linear unit (LIN, open symbols), as in Doiron et al. (2003) for $g=-1.2$. All other parameters as described in the text.

\section{Results}

We start by analyzing the model by Doiron et al. $(2003,2004)$ and demonstrate that replacing the linear summation in the model by an LIF neuron affects the behavior of this model only to a minor extent. We then explore the behavior of the model for various gain values of the inhibitory feedback loop and show that we obtain the behavior of the model by Börgers and Kopell (2003) for large feedback gains. We discuss the effect of feedback gain for both static and dynamics synapses.

Figure 2 shows the results of computer simulations for the spike train power spectral density of an excitatory neuron from the network with delayed feedback described in Figure 1 in case of fully spatially correlated $(c=1)$ and fully uncorrelated $(c=0)$ input for a feedback gain $g=-1.2$. Since the statistics of action potential firing are the same for all excitatory neurons, it suffices to show the spectra for one neuron. For uncorrelated input $(c=0)$, the last term in the expression of equation 2.5 is zero, and we obtain a spectrum that increases slightly in the range from zero to $120 \mathrm{~Hz}$. The input signal is gaussian noise with a flat spectrum, and the slight increase in spike train power spectral density reflects the dynamics of the 


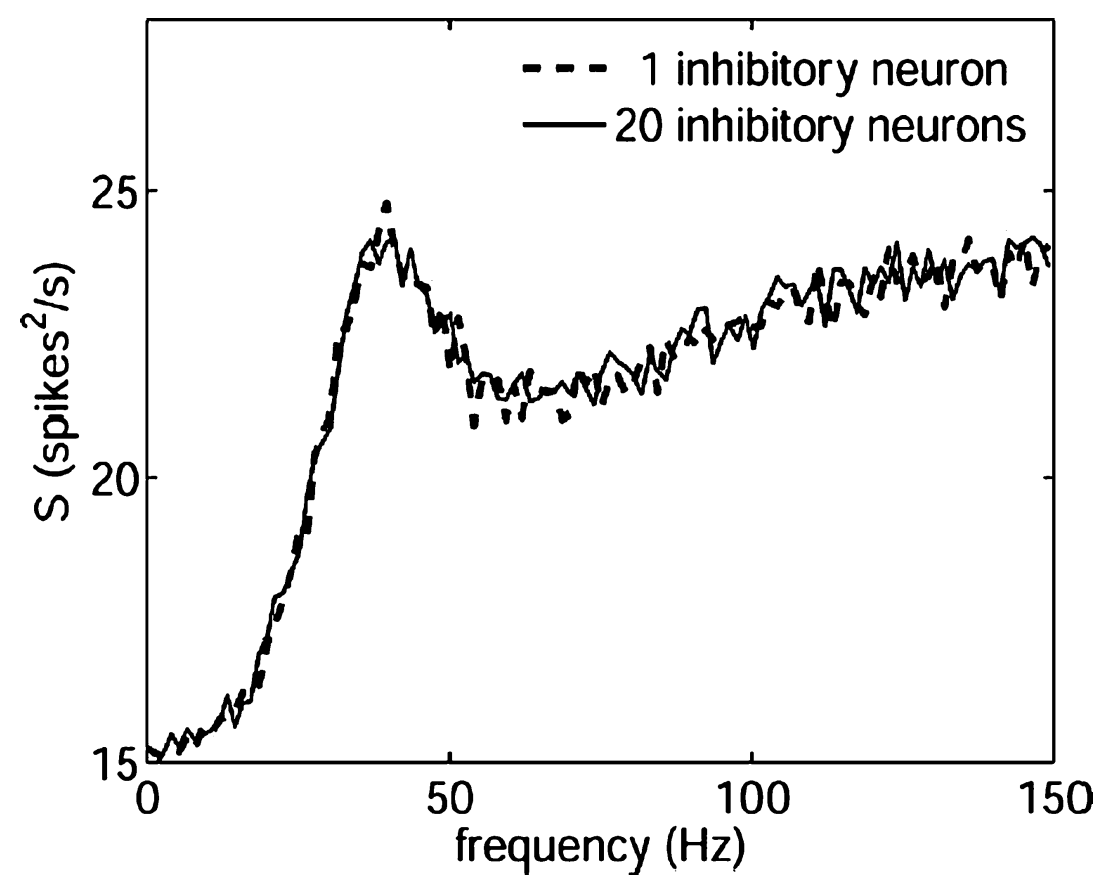

Figure 3: Spike train power spectra of an excitatory neuron for spatially correlated input when inhibition is provided by a single LIF neuron (dashed line) or a population of 20 inhibitory LIF neurons (solid line). All other parameters as in Figure 2.

excitatory LIF neurons and the effect of feedback. When the gaussian noise to the input units is fully correlated $(c=1)$, the results show a clear resonance near $40 \mathrm{~Hz}$ for the spatially correlated case. The resonance is absent in the absence of spatial correlation. For reference, the power spectrum of an excitatory neuron from the model with linear summation, as in Doiron et al. (2004), is shown for the case of fully correlated input. The peak in the power spectrum falls at a slightly lower frequency for the model with the inhibitory LIF neuron (filled circles, our model), which is due to the additional delay stemming from the dynamics of the inhibitory LIF neuron. Otherwise extensive simulations show that replacing the linear summation in the model by Doiron et al. (2004) by an LIF neuron gives very similar results, as long as the time constant of the inhibitory LIF neuron is sufficiently large (further explained in Figure 8).

The results in Figure 2 were obtained with a single inhibitory neuron, as in the study by Doiron et al. (2004). Adding more inhibitory neurons did not affect the results, as shown in Figure 3. The reason is that when the model starts to oscillate (as pointed out by Börgers and Kopell, 2003, who did their simulations with a population of inhibitory neurons), the activity of the 
inhibitory neurons synchronizes. In that case, a mean-field approximation is allowed, which explains why the population of inhibitory neurons can be replaced by a single inhibitory neuron. In further simulations, we use one inhibitory neuron.

The behavior of the network depends on the membrane time constant of the inhibitory neuron and the feedback gain. Varying these two quantities results in similar effects: a smaller membrane time constant gives rise to a higher firing rate of the inhibitory neuron, leading to a higher effective gain of the feedback. There is another effect related to the value of the membrane time constant $\tau_{m, I}$ of the inhibitory LIF neuron: when it is small, the inhibitory neuron behaves as a coincidence detector, whereas for large values of $\tau_{m, I}$, the neuron becomes an integrator (in agreement with Rudolph \& Destexhe, 2003). In the former case, the neurons spike only when there is a sufficiently large number of input spikes within a time window, small with respect to the membrane time constant. If the interval between input spikes is large relative to the membrane time constant, any effect of the single-spike inputs decays, and the neuron does not spike. The effect of the feedback gain and the membrane time constant will be discussed separately in the following.

The fact that the network oscillations in Figure 2 do not depend on power of the stimulus, differs from the results by Börgers and Kopell (2003), who report that a sufficiently large input power is required to cause oscillations. This apparent discrepancy can be understood if the feedback gain $g$ is increased (more negative values). Large values of $g$ lead to network oscillations in case of uncorrelated input (see Figure 4, left panels). Each spike of the inhibitory neuron (lower left panel) inhibits the excitatory neurons after a delay of about $15 \mathrm{~ms}$ in the feedback loop. The explanation for the apparent discrepancies with the results by Doiron et al. $(2003,2004)$ in the case of uncorrelated input is that equation 2.5 was obtained using linear response theory for the LIF neuron, as outlined by Doiron et al. (2003) and Lindner, Doiron et al. (2005). For large, synchronized inputs and when the inhibitory neuron acts like a coincidence detector, the linear response approximation for the LIF is no longer valid, and the behavior changes from that illustrated in Figure 2 into the behavior reported by Borgers and Kopell (2003). Therefore, the model in Figure 1 can reproduce the results by both Doiron et al. (2004) and Börgers and Kopell (2003) for different values of the feedback gain $g$.

It is worth stressing that the feedback gain values are not excessive or unrealistic, since the value of $|g|$ is always within the limits of the selfconsistent equation for the effective base current,

$$
\begin{aligned}
\mu_{e f f} & =\mu-g r_{0}\left(\mu_{e f f}\right), \text { with } r_{0}\left(\mu_{e f f}\right) \\
& =\left[\tau_{r}+\sqrt{\pi} \int_{\left(\mu_{e f f}-v_{T}\right) / \sqrt{D}}^{\left(\mu_{e f f}-v_{R}\right) / \sqrt{D}} e^{x^{2}} \operatorname{erfc}(x) d x\right]^{-1},
\end{aligned}
$$



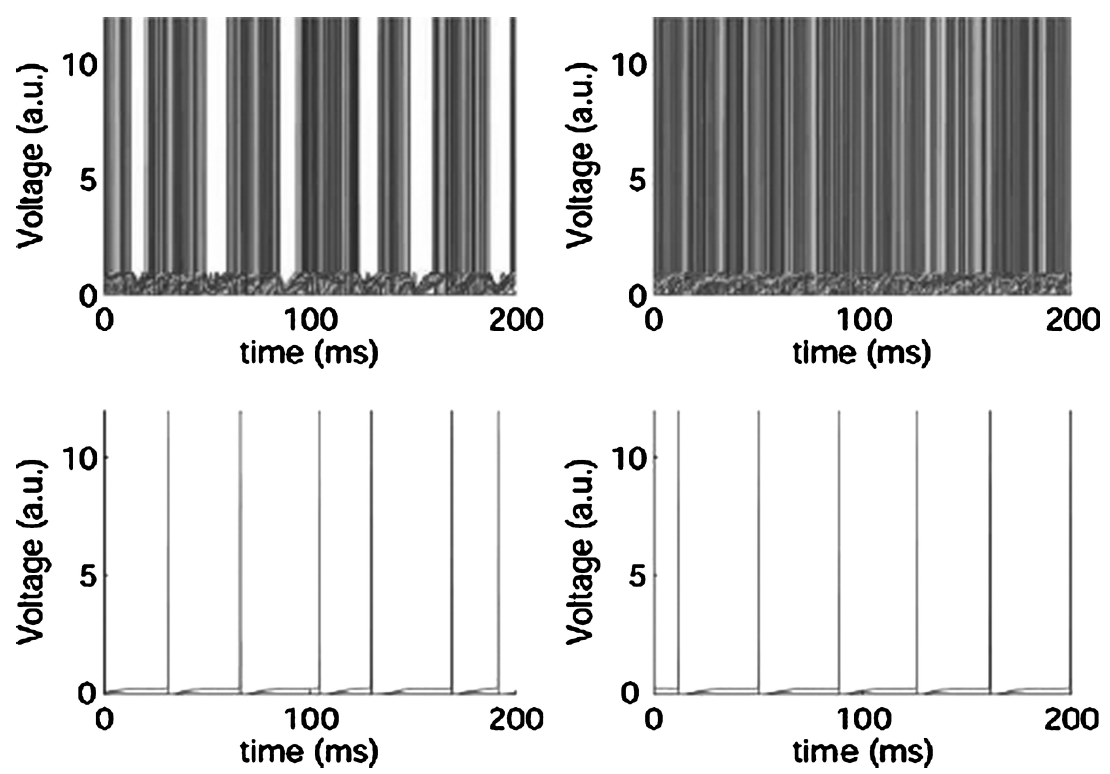

Figure 4: Superposition of spikes of all excitatory neurons (top panels) and of the inhibitory neuron (bottom panels) in case of uncorrelated gaussian white noise input with static (left) and dynamic synapses (right) for $g=-3.6$. Note that with static synapses, each action potential of the inhibitory neuron is followed by complete inhibition of the excitatory neurons after a time delay of about $15 \mathrm{~ms}$.

where $\operatorname{erfc}$ is the complementary error function. (For more detailed information about the relevance of the effective base current and the derivation of $r_{0}\left(\mu_{e f f}\right)$, see Lindner, Doiron et al. 2005.)

At this point it is useful to consider the effect of dynamic synapses. As is evident from Figure 4 (right panels), the presence of dynamic inhibitory synapses prevents complete silencing of the excitatory neurons as observed for static synapses in the case of a high feedback gain. We thus want to evaluate the effect of the feedback gain on the one hand and of the presence of dynamic synapses on the other, and explore how these two parameters change the capacity of the system to detect spatially correlated input. In order to do so, we measure the power of the spike train around the resonance frequency.

The behavior of the model is explained in more detail in Figure 5, which shows the power spectra of spiking of the excitatory neurons for the model with static synapses (left panels) and for dynamic synapses (right panels) for the case of uncorrelated gaussian white noise input ( $c=0$, upper panels) and for correlated input (lower panels, $c=1$ ) for two values of feedback 


\section{Static synapses}
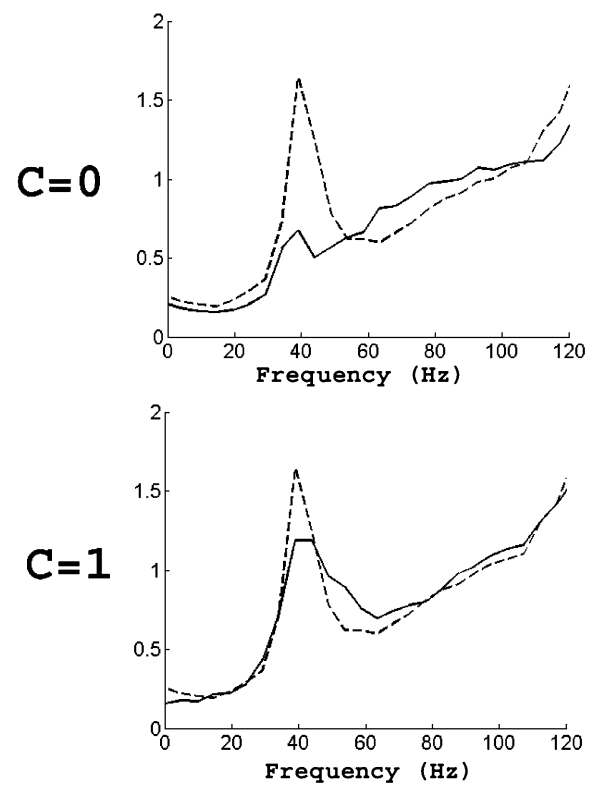

Dynamic synapses
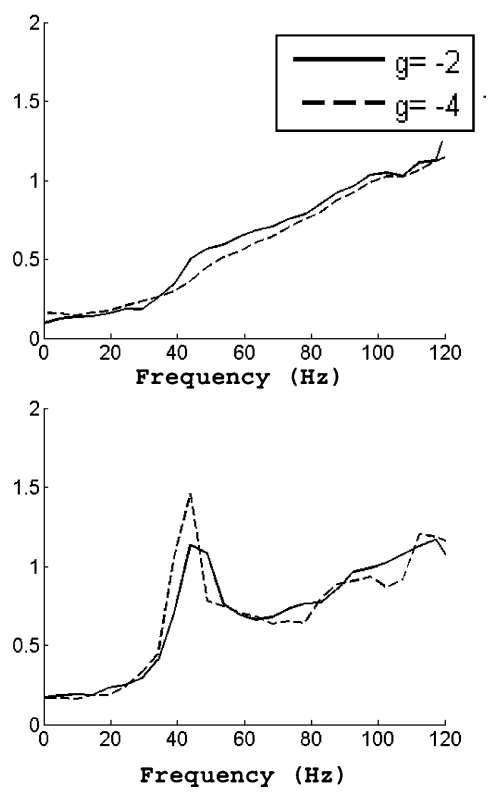

Figure 5: Spectra of the excitatory neurons for uncorrelated input ( $c=0$; upper panels) and for correlated input ( $c=1$; lower panels) for the model with static synapses (left panels) and dynamic synapses (right panels) for two different gains in the inhibitory feedback loop: $g=-2$ (solid line), $g=-4$ (dashed line). The input signal was gaussian white noise with a flat frequency spectrum up to $120 \mathrm{~Hz}$. The units along the vertical axis are in spikes ${ }^{2} / \mathrm{s}$. However, in order to compare the shape of the spectra for static and dynamic synapses and for correlated and uncorrelated input, the spectra were normalized.

gain. Variations in feedback gain cause changes in the firing rate of the excitatory and inhibitory neurons. Therefore, the power spectral density of the responses of the excitatory neurons (in spikes ${ }^{2}$ per s as in Figure 2) differs for different feedback gains. In Figures 5 and 8, we normalized the power spectral density to allow a better comparison of the shape of the spectra. The variations in firing rate are discussed later (see Figure 11).

When the input is correlated (lower panels) a resonance peak appears near $40 \mathrm{~Hz}$ for both the static and dynamic synapses for $g=-2$ and $g=$ -4 . For the uncorrelated noise with $g=-2$, the model does not show a significant resonance peak for the static or dynamic synapses. Therefore, the model is able to detect spatially correlated input for $g=-2$ with both static and dynamic synapses. However, when the feedback gain is increased to $g=-4$, the network with static synapses resonates also in the absence of 


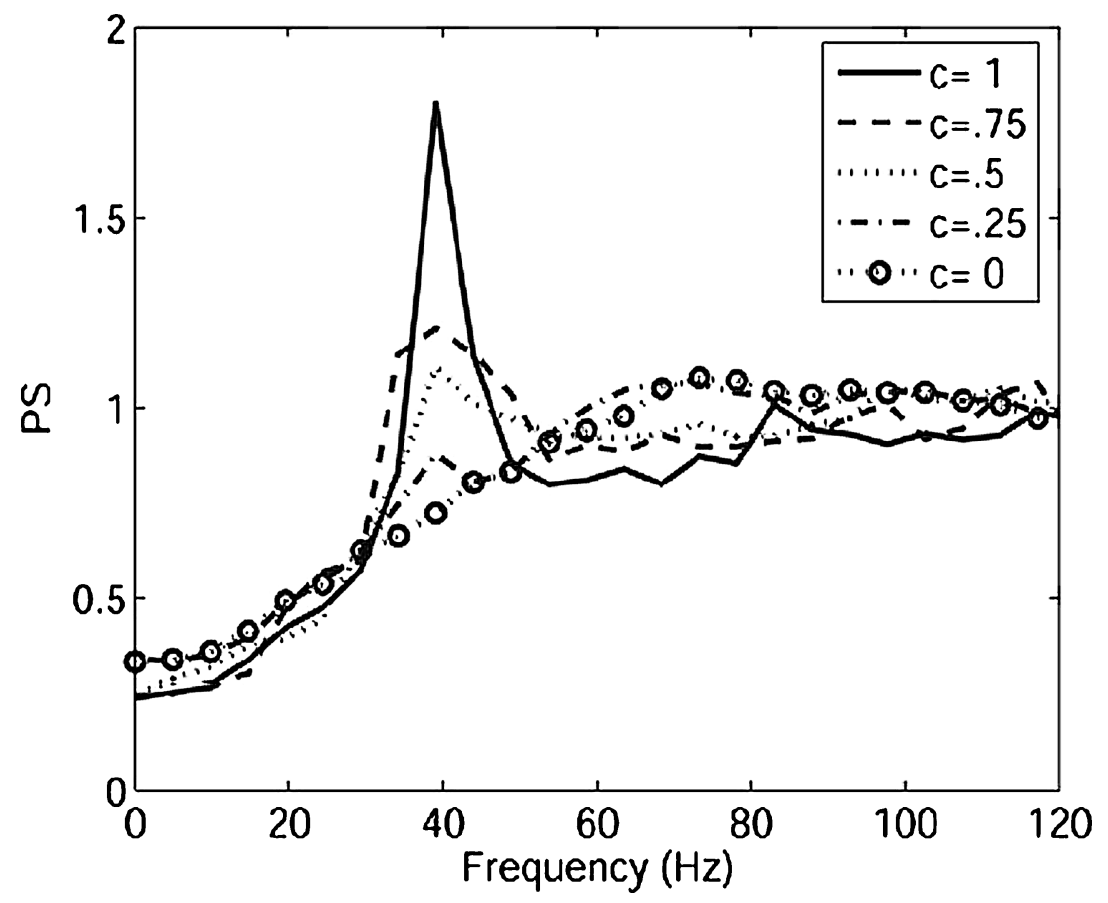

Figure 6: Power spectra of the excitatory neurons for the model with dynamic synapses for different values of correlated input. Gains in the inhibitory feedback loop: $g=-2$. The input signal was gaussian white noise with a flat frequency spectrum up to $120 \mathrm{~Hz}$. In order to compare the shape of the spectra, the units along the vertical axis were normalized.

spatially correlated input (upper left panel). These results demonstrate that we can reproduce the results of Doiron et al. $(2003,2004)$ for static synapses as long as the feedback gain is relatively small and obtain the results of Börgers and Kopell (2003) for relatively high feedback gains. When dynamic synapses are included in the model, the model behavior does not depend on feedback gain, since there is no resonance for physiological feedback gains when the input is uncorrelated (upper-right panel). If the input is correlated, there is a peak near $40 \mathrm{~Hz}$ as soon as there is feedback in the model.

For simplicity, we have considered only the extreme cases of fully correlated $(c=1)$ or fully uncorrelated $(c=0)$ input in Figure 5 . Values of $c$ in the range between 0 and 1 yield results that are intermediate between the two extremes described above. This is illustrated in Figure 6, which clearly illustrates that the resonance peak decreases with decreasing values of $c$. Notice that for $c=1$ we find a peak near $40 \mathrm{~Hz}$, but a decrease in the power 
spectrum in the range between 50 and $80 \mathrm{~Hz}$. This can be understood by the fact that the term $g e^{-i \omega \tau_{D}} K_{\tau}^{E}(\omega) K_{\tau}^{I}(\omega) A^{E}(\omega) A^{I}(\omega)$ in the denominator in the last term in equation 2.5 contains a factor $e^{-i \omega \tau_{D}}$ related to the time delay $\tau_{D}$ in the feedback loop. When the term $g e^{-i \omega \tau_{D}} K_{\tau}^{E}(\omega) K_{\tau}^{I}(\omega) A^{E}(\omega) A^{I}(\omega)$ is maximal, it causes a peak in the spectrum near $40 \mathrm{~Hz}$. For higher frequencies, the term $e^{-i \omega \tau_{D}}$ changes sign, which, together with the frequency dependence of the susceptibility $A$ and synaptic transfer function $K$, changes the sign of $g e^{-i \omega \tau_{D}} K_{\tau}^{E}(\omega) K_{\tau}^{I}(\omega) A^{E}(\omega) A^{I}(\omega)$, causing a decrease in the spectrum.

For our choice of time constants (membrane time constant $6 \mathrm{~ms}$ for excitatory and inhibitory neurons), there will or will not occur a resonance as indicated by the peak in Figure 2 depending on the strength of the feedback coupling, the characteristics of the input signal, and the dynamics of the synapse. The amplitude of the resonance can be quantified introducing the statistics $\Gamma_{f_{1}, f_{2}}=\int_{f_{1}}^{f_{2}} S(f) d f$, where the power spectrum of the spike train of the excitatory neurons is represented by $S(f)$. As in Doiron et al. $(2003,2004)$, we quantify the resonance by $\Gamma_{F B}=\Gamma_{30,50}-\Gamma_{2,22}$, comparing the value of $\Gamma$ near the resonance frequency to the value of $\Gamma$ in a low-frequency region.

Figure 7 (upper panel) shows that for relatively small values of the feedback gain, the value $\Gamma_{F B}$ increases for small values of $g(-2<g<0)$ for fully correlated input to the excitatory neurons for both the static and dynamic synapses, indicating increasing peak values of oscillations in the presence of fully correlated input. However, $\Gamma_{F B}$ remains small for uncorrelated input as long as $-2<g<0$. For $g<-2$, the network also starts to oscillate for uncorrelated input $(c=0)$ for the case of static synapses. The spike train power near the resonance frequency rapidly reaches the same value as that for the correlated input case. This is not the case for the model with dynamic synapses, which shows a very robust behavior for both uncorrelated and correlated input over the whole range of feedback gains studied.

To quantify this situation even better, we introduce a discrimination index,

$$
\rho_{0-1}=\frac{\Gamma_{F B}(c=1)-\Gamma_{F B}(c=0)}{\Gamma_{F B}(c=1)+\Gamma_{F B}(c=0)} .
$$

This index indicates to what extent the network is able to distinguish between a fully spatially correlated and a fully uncorrelated input. The lower panel of Figure 7 shows that dynamic synapses (filled symbols) guarantee a clear and constant discrimination for all feedback gain values, whereas the model with static synapses (open symbols) detects the correlated input only at intermediate feedback gains (range from -1 to -2 ) but cannot distinguish correlated and uncorrelated inputs for feedback gains $g<-2$.

It has been shown (Whittington, Traub, \& Jeffreys, 1995; Traub, Whittington, Colling, Buzsaki, \& Jeffreys, 1996) that gamma oscillations in hippocampal interneurons are sensitive to the decay time constant of the 

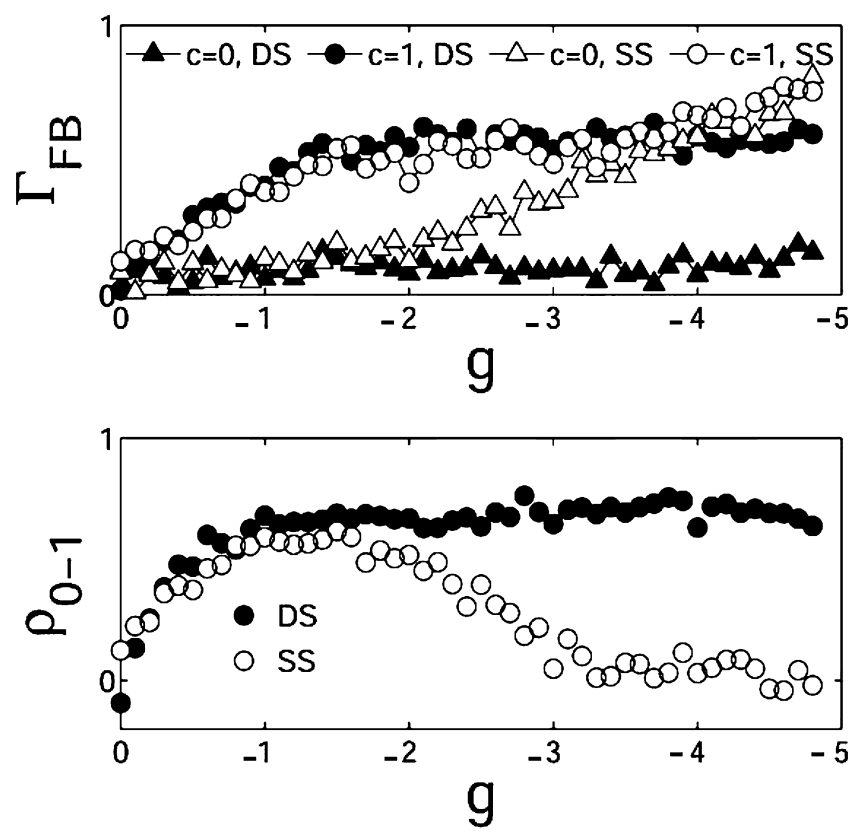

Figure 7: (Top) Power $\Gamma_{F B}=\Gamma_{30,50}-\Gamma_{2,22}$ near the resonance peak $(30-50 \mathrm{~Hz})$ relative to that in the frequency range between 2 and $22 \mathrm{~Hz}$ in the presence of correlated ( $c=1$; circles) and uncorrelated ( $c=0$; triangles) input, with static synapses (SS; open symbols) and dynamic synapses (DS; filled symbols). (Bottom) Ability to discriminate correlated and uncorrelated input $\rho_{0-1}$ (see equation 3.2) for static (SS, open symbols) and dynamic (DS, closed symbols) synapses as a function of the inhibitory gain.

$\mathrm{GABA}_{A}$ synapse in the sense that the oscillation frequency decreases for increasing values of that time constant. This is compatible with the fact that the oscillation frequency in the models by Börgers and Kopell (2003) and by Doiron et al. $(2003,2004)$ depends on the effective time delay in the feedback loop, which includes the time delay (absent in the Börgers and Kopell model), the dynamics of the synapses, and the dynamics of the inhibitory LIF neurons. Since changing the time constant of the inhibitory LIF neuron changes both the effective delay in the feedback loop and the behavioral characteristics of the LIF neuron (integrator versus coincidence detector), we have analyzed the behavior of the model for various time constants $\tau_{m, I}$ for the inhibitory LIF neuron.

As mentioned before, the neurons in the network start to oscillate with synchronized firing of the excitatory neurons when the membrane time constant of the inhibitory neuron is small. This synchronization is independent of the nature of the external stimulus as long as the intensity is 


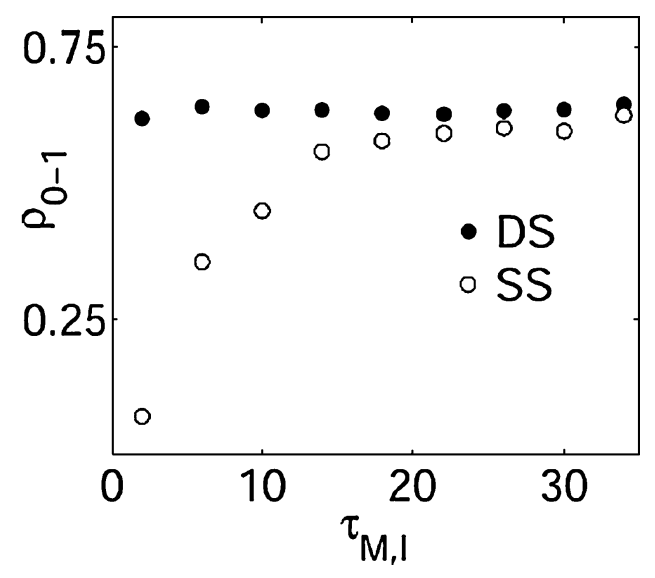

Figure 8: Ability to discriminate correlated and uncorrelated input $\rho_{0-1}$ (see equation 3.2) for static (SS, open symbols) and dynamic (DS, filled symbols) synapses versus the membrane time constant of the inhibitory neuron, with $g=-1.2$.

sufficiently large and when the time constant $\tau_{m, I}$ is small (see Figure 8). For time constants larger than $15 \mathrm{~ms}$, the linear approximation of the inhibitory LIF neuron is valid, and the network behaves as in Doiron et al. $(2004,2004)$. When dynamic synapses are included in the model, the separation between correlated and uncorrelated input is also preserved for small values of $\tau_{m, I}$; the index $\rho_{0-1}$, which represents the ability to discriminate between correlated and uncorrelated input, is constant for all values of the membrane time constant $\tau_{m, I}$.

Another novel aspect of the model with dynamic synapses relates to the ability to preserve the frequency content of the input signal. In the study by Börgers and Kopell (2003), the oscillation frequency depends on only the effective time delay in the feedback loop between excitatory and inhibitory neurons and is present as long as the power of the input signal is sufficiently large. In the model by Doiron et al. (2003), the presence of oscillations depends on the presence of spatially correlated input, and the oscillation frequency depends on the effective delay in the feedback loop, not the temporal properties of the input. With dynamic synapses, the system also preserves the temporal information contained in the input. This is illustrated in Figure 9, which shows the spectra of the spike responses of the excitatory neurons in response to bandpass filtered gaussian noise with a center frequency at $60 \mathrm{~Hz}$ - a frequency well above the resonance peak near $40 \mathrm{~Hz}$. The bandpass filtered noise is obtained by filtering gaussian white noise with a flat spectrum up to $150 \mathrm{~Hz}$ with a second-order Butterworth bandpass filter with passband between 53 and $67 \mathrm{~Hz}$. After filtering in the 
Static synapses

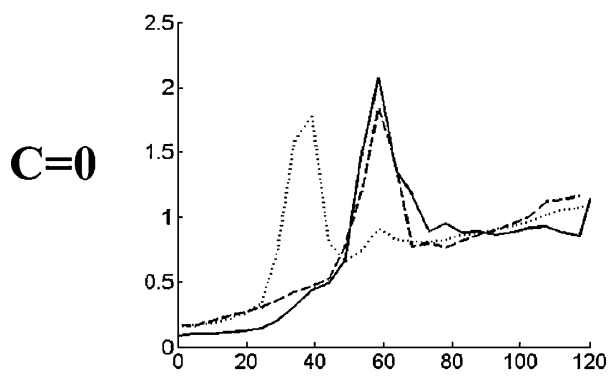

Frequency $(\mathrm{Hz})$

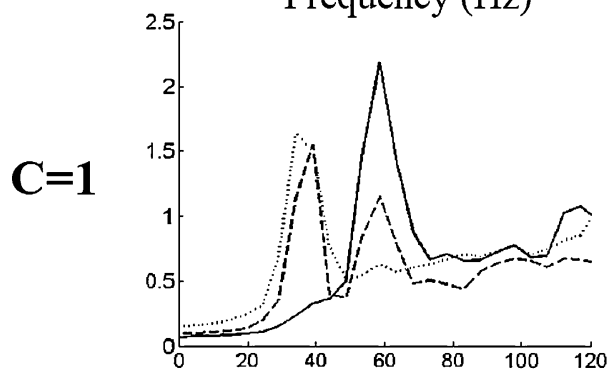

Frequency $(\mathrm{Hz})$
Dynamic synapses

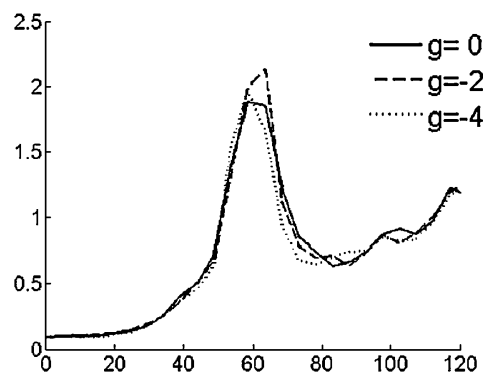

Frequency $(\mathrm{Hz})$

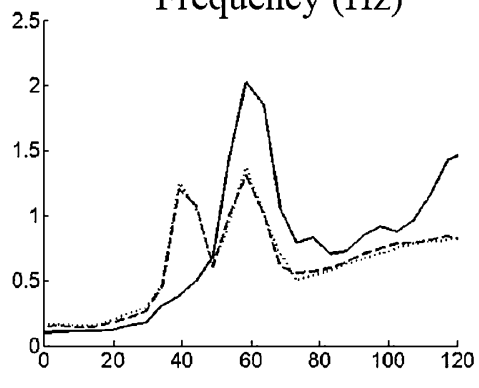

Frequency $(\mathrm{Hz})$

Figure 9: Spectra of the excitatory neurons for uncorrelated input $(C=0$; upper panels) and for correlated input ( $C=1$; lower panels) for the model with static synapses (left panels) and dynamic synapses (right panels) for three different gains in the inhibitory feedback loop: $g=0$ (solid line), $g=-2$ (dashed line) and $g=-4$ (dotted line). The input signal was bandpass filtered gaussian white noise, centered at $60 \mathrm{~Hz}$. The units along the vertical axis are in spikes ${ }^{2} / \mathrm{s}$. In order to compare the shape of the spectra, the spectra were normalized.

forward direction, the filtered sequence is reversed to ensure a zero-phase shift. The power of the bandpass filtered signal is normalized such that the power of the input signal is the same in all conditions.

Without feedback $(g=0)$ the solid lines show the spectra of the responses to the bandpass filtered noise with a clear peak near $60 \mathrm{~Hz}$. For the static synapses (left-hand panels), the amplitude of the peak near $60 \mathrm{~Hz}$ decreases with increasing feedback gain. If the input is spatially correlated, a resonance peak appears near $40 \mathrm{~Hz}$, consistent with the results of Doiron et al. $(2003,2004)$ and with the results in Figure 5 and Figure 7 , which show that a resonance peak is found for intermediate feedback gains only if the input is correlated. If the bandpass filtered noise at the input is spatially uncorrelated ( $c=0$; upper left panel), a peak near $40 \mathrm{~Hz}$ also appears for 
the high feedback gain of -4 , in agreement with our previous results (see Figure 5). If the bandpass filtered noise for the excitatory input units is spatially correlated ( $c=1$; lower left panel), the peak near $60 \mathrm{~Hz}$ disappears and a peak near $40 \mathrm{~Hz}$ appears, for a feedback gain of -4 .

The spectra of the spike output of the excitatory neurons for the model with dynamic synapses differ from those for the static synapses in two aspects. The first difference is that the spectra for the model with dynamic synapses are almost identical for the uncorrelated bandpass filtered noise for all feedback gain values ( $c=0$, upper right panel). The almost identical shape of the spectra reflects the robustness of the model behavior with dynamic synapses. If the bandpass filtered noise is correlated $(c=1$, lower right panel), a peak appears near $40 \mathrm{~Hz}$, but in addition, the peak at the input near $60 \mathrm{~Hz}$ is preserved. This finding reflects that the model with the dynamic synapses has two important advantages over the model with static synapses: the responses of the excitatory neurons are robust for changes in feedback gain and the spectra preserve the spectra of the input, in addition to the peak in the spectrum indicating correlated input.

In order to explore the effect of the feedback gain on the spike responses near the input frequency, we have explored the ratio $\frac{\Gamma_{52.5 .67 .5}}{\Gamma_{72.5 .87 .5}}$, which relates the power of the excitatory neurons near the bandpass filtered input at $60 \mathrm{~Hz}$ to the value of power near $80 \mathrm{~Hz}$, which is in a region where the power spectrum is not affected by feedback or resonance. A high value for this ratio reflects large response amplitudes near the input frequency of $60 \mathrm{~Hz}$, whereas small values indicate that the input signal is not present in the spike responses. With feedback gain equal to zero, there is no difference between static and dynamic synapses (see Figure 10). As illustrated in Figures 5, 7, and 9, the ability to discriminate between spatially correlated and uncorrelated input is about the same for small feedback gains for dynamic and static synapses. For the range of feedback values between -1 and -2 , we reproduce the behavior reported by Doiron et al. (2004) for static synapses; there is a resonance peak for spatially correlated input (see Figure 9, lower left panel), but the characteristics of the input signal are almost lost. This explains why for the static synapses, the ratio $\frac{\Gamma_{52.5,67.5}}{\Gamma_{72.5,87.5}}$ is smaller in the range $-2<g<-1$ than for $g=0$. For values of $g<-2$, the system starts to oscillate at $40 \mathrm{~Hz}$ for the static synapses regardless of spatial correlation of the input (see Figures 5, 7, and 9), which explains the decrease of $\frac{\Gamma_{52.5,67.5}}{\Gamma_{72.5 .87 .5}}$ to values near 1 for static synapses. Quite remarkably, the ratio $\frac{\Gamma_{52.5 .67 .5}}{\Gamma_{72.5 .87 .5}}$ remains approximately constant for the dynamic synapses for all values of $g$, indicating a preservation of the input characteristics in the firing rate of the excitatory neurons.

The robustness of the model responses with the dynamic synapses is reflected not only in the spectra (see Figures 8,9, and 10) but also in the firing rates of the excitatory and inhibitory neurons in the model. This is illustrated in Figure 11, which shows the firing rate of the excitatory (E) and inhibitory 


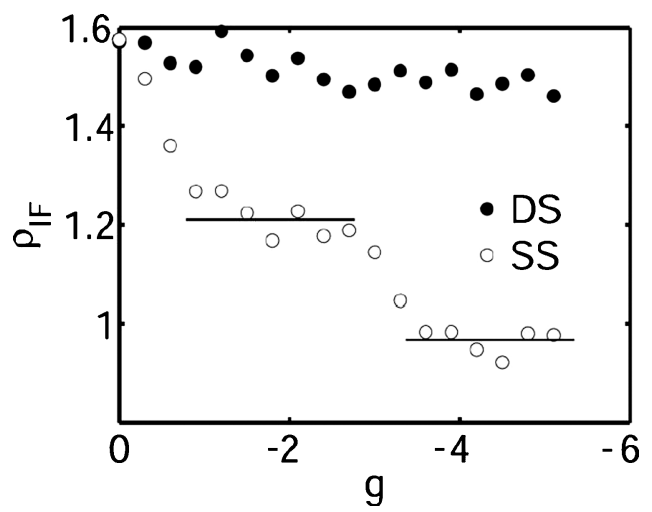

Figure 10: Discrimination index $\rho_{I F}$ to discriminate the input frequency near $60 \mathrm{~Hz}$ in the responses of the excitatory neurons, defined as the power around $60 \mathrm{~Hz}$ divided by the power around $80 \mathrm{~Hz}\left(\frac{\Gamma_{52.5,67.5}}{\Gamma_{72.5,87.5}}\right)$ for static (open symbols, SS) and dynamic (filled symbols, DS) synapses.

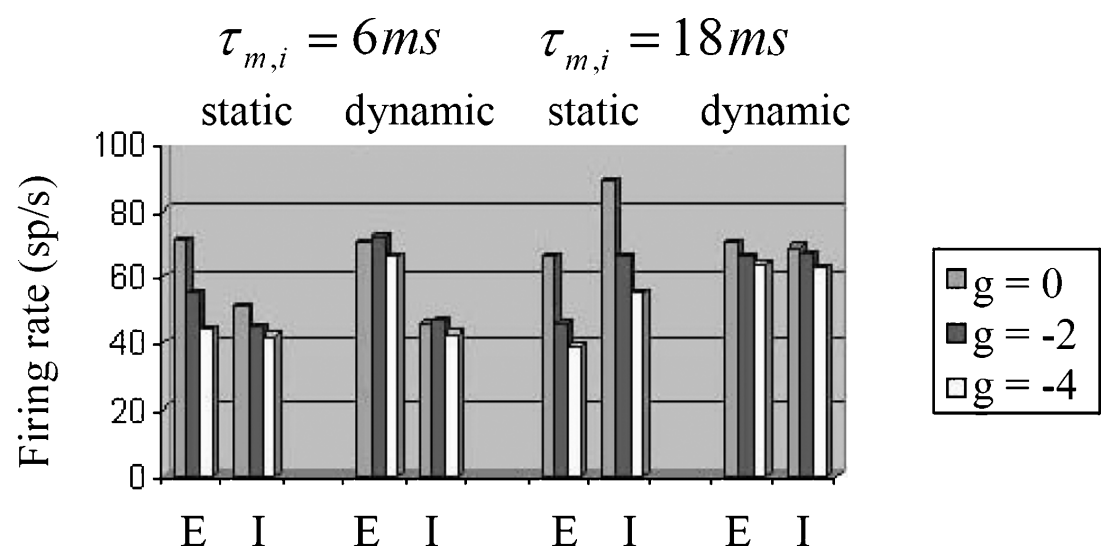

Figure 11: Firing rate of excitatory (E) and inhibitory (I) neurons for the models with static and dynamic synapses. Data at left (right) hand correspond to model with inhibitory LIF neuron with leak time constant of 6 (18) ms. Except for differences in time constant of inhibitory neuron, static versus dynamic synapses and feedback gain, all other parameters were the same for all conditions.

(I) neurons for the models with static and dynamic synapses for the various feedback gains and for two different time constants of the inhibitory neurons (6 and $18 \mathrm{~ms}$ ). For the static synapses, the firing rate of the excitatory neurons decreases with increasing feedback gain. The difference in firing rate for $g=0$ and $g=-4$ is typically about $30 \%$. A similar decrease in firing 
rate is observed for the inhibitory neurons. However, for the model with dynamic synapses, the firing rates of the excitatory and inhibitory neurons depend only weakly on the feedback gain. Note that this phenomenon is independent of the time constant of the inhibitory neurons.

\section{Discussion}

The main results of this study can be summarized in two conclusions. The first is that the different results, reported by Börgers and Kopell (2003) and by Doiron et al. $(2003,2004)$, who used models with the same architecture but with a qualitatively different behavior, can be explained by the responses of a single model with different feedback gains. The simulations presented here show that the same architecture with pools of excitatory and inhibitory neurons can display a different behavior when the parameter values are in a different range. For small and intermediate feedback gains, the model starts to oscillate if the input to the excitatory input units is spatially correlated. Without correlation, the resonance peak in the spike responses is absent. The resonance in the model, as reported by Börgers and Kopell (2003), which appears as soon as the input to the excitatory neurons is sufficiently large (regardless of correlation between input to the neurons), is obtained if the feedback gain is sufficiently large, in agreement with the conditions for resonance in their study. Another difference between the models by Börgers and Kopell (2003) and Doiron et al. $(2003,2004)$ is that the latter has only one inhibitory unit, whereas Börgers and Kopell have a population of inhibitory neurons. In this study we have shown that the behavior of the model by Doiron et al. $(2003,2004)$ does not change if the linear unit, which sums the spikes of the excitatory neurons and which feeds the feedback loop, is replaced by an LIF neuron. Figure 3 shows that the results are not affected either, when several inhibitory neurons are added in parallel. Börgers and Kopell (2005) also report that the qualitative behavior of the model is the same if several inhibitory neurons are added. In their study, suppression of excitatory cells can occur for asynchronously firing inhibitory cells. In our study, the inhibitory cells fire in synchrony for spatially correlated input $(c=1)$ and for high feedback gains. For $c=0$ and for small feedback gains, the inhibitory neurons in our model do not fire in synchrony, and neither do the excitatory cells. However, if there are several inhibitory neurons, the network models starts oscillations at slightly higher feedback gains than for a single inhibitory neuron. In summary, the behavior of the model is qualitatively the same for a single inhibitory neuron and for the case of several inhibitory neurons.

The second conclusion of our study is that replacing synapses with a constant efficacy by dynamic synapses has two major implications: the model behavior becomes robust for changes in feedback gain and in time constant of the inhibitory LIF neuron. The resonance peak appears only for correlated input stimuli. Moreover, the model with dynamic synapses preserves 
the characteristics of the input in the spike responses. From a biological perspective, this is highly relevant since it allows transmission of the temporal properties of the input signal while the resonance provides a label to indicate whether the input is spatially correlated. In visual cortex cells, responses reflect the temporal properties of the stimulus in the receptive field, but in addition can reveal gamma-frequency components (see, e.g., Reynolds \& Desimone, 1999; Roelfsema, Lamme, \& Spekreijse, 2004). In addition, several studies (e.g., Baker, Pinches, \& Lemon, 2003; Schoffelen et al., 2005) have shown that multiple EEG rhythms can occur simultaneously, which is in contradiction with the behavior of some models in the literature (see, e.g., Börgers \& Kopell, 2003; Doiron et al., 2004), which reveal oscillations only at one particular frequency.

The finding that the activity of the network can reflect both the temporal characteristics of the input signal (frequency of input modulations), which drives the network, and synchronous oscillations at one particular frequency is new. Previous studies modeling neurons as coupled oscillators have shown that when the width of the distribution of the intrinsic frequencies is small compared to the coupling strength, all oscillators become phase-locked (Ariaratnam \& Strogatz, 2001). When the distribution of intrinsic frequencies becomes larger, the network can reveal a broad spectrum of dynamics, such as frequency locking at a single frequency, incoherent firing with a broad range of frequencies, oscillator death, and hybrid solutions that combine two or more of these states, depending on the coupling strength. Tsodyks, Mitkov, and Sompolinsky (1993) studied a system of globally coupled oscillators with pulse interactions (mimicking coupling by presynaptic action potentials). In such a system, the completely phase-locked state is unstable to weak disorder. For a small inhomogeneity, the oscillators are divided in two populations, one of which exhibits phaselocked periodic behavior, whereas the other consists of unlocked oscillators, which exhibit aperiodic patterns that are only partially synchronized. In this context it is important to stress, that the spectra shown in Figures 5, 6, and 9 are representative for all excitatory neurons. That is, the oscillations and the input characteristics can be found in each neuron and do not reflect a partitioning within the pool of excitatory neurons.

The novel finding that a group of neurons can code both the correlation between parallel neuronal input channels and the temporal characteristics of input signals is relevant for several reasons. As explained in section 1, the phenomenon of oscillations in neuronal activity in groups of neurons has long been known. However the functional significance is yet the subject of speculation (see, e.g., Fries, 2005). Moreover, the neuronal mechanisms, which are responsible for the initiation of oscillatory activity, are barely known. It is well known that cells in visual cortex can fire in synchrony at frequencies near 40 to $60 \mathrm{~Hz}$ and at the same time can reproduce the temporal dynamics of changes in the light in the receptive field. For example, Fries, Reynolds, Rorie, and Desimone (2001) report typical changes in firing 
rate at a latency of about $25 \mathrm{~ms}$ in cat primary visual cortex, related to onset and offset of a visual stimulus in the receptive field of these neurons. At the same time, they find a correlated oscillatory activity at frequencies in the range between 40 and $70 \mathrm{~Hz}$. This clearly illustrates that the activity of cells in cat primary visual cortex reflects simultaneously temporal changes in light falling in the receptive field as well as correlated oscillatory activity at 40 to $70 \mathrm{~Hz}$. Similarly, cells in visual areas V2 and V4 reflect the dynamics of stimuli in the receptive field, but at the same time reveal correlated activity in the $\gamma$-range (Reynolds \& Desimone, 1999). As far as we know, our study is the first to show that the temporal dynamics of activity in a population of neurons can reflect both changes in the stimulus that drives the cells and oscillatory activity.

van Vreeswijk and Hansel (2001) studied the emergence of synchronized burst activity in networks of neurons with spike adaptation. They showed that networks of tonically firing adapting excitatory neurons can evolve to a state where the neurons burst in a synchronized manner. These authors showed that synchronized bursting is robust against inhomogeneities, sparseness of the connectivity, and noise. In networks of two populations, one excitatory and one inhibitory, they found that decreasing the inhibitory feedback can cause the network to switch from a tonically active, asynchronous state to the synchronized bursting state. In our study, we had dynamic synapses instead of spike adaptation, as in the study by van Vreeswijk and Hansel (2001). Another difference is that the latter study did not consider external input but studied tonically active neurons instead. Our study therefore is a further extension of the work of van Vreeswijk and Hansel by showing the effect of external input on the stable states of a network with excitatory and inhibitory neurons coupled by dynamic synapses.

In the model shown in Figure 1, the dynamic synapses were implemented in the feedback (i.e., the $I \rightarrow E$ ) path. If the firing rate of the inhibitory neuron increases, the efficacy of the dynamic synapses decreases, which acts like an automatic gain control. This explains partly why the responses of the model with dynamic synapses hardly depend on the feedback loop gain. This finding is in agreement with Pantic et al. (2003), who demonstrated that dynamic synapses can perform good coincidence detection over a large range of frequencies for one suitably chosen threshold value, whereas static synapses require an adaptive frequency-dependent threshold value for good detection. In fact, the adaptation is done by the synapses themselves. Dynamic synapses in the feedforward loop have also been discussed in several studies. The common message of these studies (see, e.g., Natschlager, Maas, \& Zador, 2001; Senn, Segev, \& Tsodyks, 1998; Pantic et al., 2003) is that dynamic synapses in the $E \rightarrow I$ path are very effective in allowing a correct coincidence detection for a wide range of firing rates of the excitatory neurons without changing the base current to the inhibitory neurons. 
Initially it came as a surprise that the model shown in Figure 1 revealed oscillations also for uncorrelated noise $(c=0)$ for large feedback gains. The last term in equation 2.5 is zero for $c=0$. The explanation for the unexpected resonance is that equation 2.5 was derived using linear-response theory (see Doiron et al., 2004, and Lindner, Chacron, \& Longtin, 2005, where the assumptions and mathematical analyses are explained in detail). However, the linear approximations of an LIF neuron are valid only for relatively long time constants of the neuron, where it operates mainly as an integrator. This approximation is no longer valid when the time constant of the LIF neuron is short, transforming the neuron into a coincidence detector. Similarly, a high-feedback gain gives rise to full inhibition of the excitatory neurons for each action potential of the inhibitory neuron (see Figure 4, left panel), followed by a release of excitatory output. The large inhibitory input to the excitatory neurons synchronizes the excitatory neurons (precisely as in Börgers \& Kopell, 2003), leading to a burst of activity to the inhibitory neuron. For such volleys of synchronized input, the linear approximation for the LIF neuron also fails.

\section{Appendix}

This appendix gives a derivation of equation 2.5. It mainly follows the lines set out in Doiron et al. (2004) and in Lindner, Chacron et al. (2005) and Lindner, Doiron et al. (2005).

The input to the excitatory neurons is split into two parts. The first part consists of the base current $\mu$, the internal neuron-specific noise $\eta_{i}(t)$, and the time-independent mean of the feedback $\left(g\left\langle S T_{I}(t)\right\rangle\right.$ with $\left.g<0\right)$ from the inhibitory neuron to the excitatory neuron. The second part consists of the external input $\sigma\left\lfloor\sqrt{1-c} \xi_{j}(t)+\sqrt{c} \xi_{G}(t)\right\rfloor$ and the time-dependent part of the feedback from the inhibitory neurons.

Using a linear response approximation for a leaky integrate-and-fire neuron with susceptibility $A^{E}(\omega)$ and $A^{I}(\omega)$ (see Lindner \& SchimanskyGeier, 2001; Lindner, Schimansky-Geier, \& Longtin, 2002) for the excitatory neurons and inhibitory neurons, respectively, the spectrum of the spike train of the excitatory neuron is given by

$$
s_{j}^{E}(\omega)=s_{0, j}^{E}(\omega)+A^{E}(\omega)\left[I_{j}(\omega)+\frac{g}{N} e^{-i \omega \tau_{D}} K_{\tau}^{I}(\omega) s^{I}(\omega)\right],
$$

where $s_{j}^{E}(\omega)$ and $s_{0, j}^{E}(\omega)$ represent the spike train of the $j$ th excitatory neuron in the presence and absence, respectively, of the external stimulus and feedback. $K_{\tau}^{I}(\omega)$ represents the postsynaptic dynamics for the synapses from the inhibitory to the excitatory neurons as defined in equation 2.4 in the frequency domain, and $A^{E}(\omega)$ and $A^{I}(\omega)$ represent the susceptibility in the frequency domain with respect to the input of the excitatory neurons and the inhibitory neurons, respectively (for details, see Doiron et al., 2004; 
Lindner \& Schimansky-Geier, 2001; Lindner et al., 2002). The term $e^{-i \omega \tau_{D}}$ is the Fourier representation of the time delay in the inhibitory feedback loop. For the activity of the inhibitory neuron in the frequency domain, we obtain

$$
s^{I}(\omega)=A^{I}(\omega) \mathrm{K}_{\tau}^{E}(\omega) \sum_{j=1}^{N_{I}} s_{j}^{E}(\omega) .
$$

Inserting equation A.2 in equation A.1 and with $I_{j}(\omega)$ as the Fourier transform of $I_{j}(t)=\mu+\eta_{j}(t)+\sigma\left\lfloor\sqrt{1-c} \xi_{j}(t)+\sqrt{c} \xi_{G}(t)\right\rfloor$ gives for the power spectrum of the spike train of the $j$ th excitatory neuron

$$
\begin{aligned}
\left\langle\tilde{s}_{j}^{E} \tilde{s}_{j}^{*^{E}}\right\rangle= & \left\langle\tilde{s}_{0, j}^{E} \tilde{s}_{0, j}^{*^{E}}\right\rangle+\left\langle\tilde{A}^{E} \tilde{I}_{j} \tilde{s}_{0, j}^{*^{E}}\right\rangle+\left\langle\tilde{A}^{* E} \tilde{I}_{j}^{*} \tilde{s}_{0, j}^{E}\right\rangle \\
& +\left\langle\tilde{s}_{0, j}^{E} \tilde{A}^{*^{E}} \frac{g}{N} e^{i \omega \tau_{D}} \tilde{K}_{\tau}^{I^{*}} \tilde{A}^{I^{*}} \tilde{K}_{\tau}^{E^{*}} \sum_{k=1}^{N_{I}} \tilde{s}_{k}^{E^{*}}\right\rangle \\
& +\left\langle\tilde{s}_{0, j}^{E^{*}} \tilde{A}^{E} \frac{g}{N} e^{-i \omega \tau_{D}} \tilde{K}_{\tau}^{I} \tilde{A}^{I} \tilde{K}_{\tau}^{E} \sum_{k=1}^{N_{I}} \tilde{s}_{k}^{E}\right\rangle+\left\langle\left|\tilde{A}^{E}\right|^{2}\left|\tilde{I}_{j}\right|^{2}\right\rangle \\
& +\left\langle\left|\tilde{A}^{E}\right|^{2} \tilde{I}_{j} \frac{g}{N} e^{i \omega \tau_{D}} \tilde{K}_{\tau}^{I^{*}} \tilde{A}^{I^{*}} \tilde{K}_{\tau}^{E^{*}} \sum_{k=1}^{N_{I}} \tilde{s}_{k}^{E^{*}}\right\rangle \\
& +\left\langle\left|\tilde{A}^{E}\right|^{2} \tilde{I}_{j}^{*} \frac{g}{N} e^{-i \omega \tau_{D}} \tilde{K}_{\tau}^{I} \tilde{A}^{I} \tilde{K}_{\tau}^{E} \sum_{k=1}^{N_{I}} \tilde{s}_{k}^{E}\right\rangle \\
& +\left\langle\left|\tilde{A}^{E}\right|^{2}\left(\frac{g}{N}\right)^{2}\left|\tilde{K}_{\tau}^{I}\right|^{2}\left|\tilde{A}^{I}\right|^{2}\left|\tilde{K}_{\tau}^{E}\right|^{2} \sum_{k, l}^{N_{E}} \tilde{s}_{k}^{E} \tilde{s}_{l}^{E^{*}}\right\rangle,
\end{aligned}
$$

where $\tilde{x}$ represents the Fourier transform of $x$.

Using that $\left\langle s_{0, j}^{E}(\omega) s_{k}^{*^{E}}(\omega)\right\rangle=\left\langle s_{0, j}^{E}(\omega) I_{j}^{*}(\omega)\right\rangle=\left\langle s_{j}^{E}(\omega) \xi_{k}^{*}(\omega)\right\rangle=$ $\left\langle\xi_{G}(\omega) \xi_{k}^{*}(\omega)\right\rangle=0$ for $j \neq k$ explains why the second and third terms on the right-hand side of the equal sign are equal to zero.

Substitution of equation A.2 in A.1 and solving for $\sum_{j=1}^{N_{I}} s_{j}^{E}(\omega)$ gives

$$
\sum_{k=1}^{N_{E}} \tilde{s}_{k}^{E}=\frac{\sum_{j=1}^{N_{E}} \tilde{s}_{0, j}^{E}+\tilde{A}^{E} \sum_{j=1}^{N_{E}} \tilde{I}_{j}}{1-\tilde{A}^{E} g e^{-i \omega \tau_{D}} \tilde{K}_{\tau}^{I} \tilde{A}^{I} \tilde{K}_{\tau}^{E}} .
$$


Substitution of equation A.4 in A.3 and using that $\left\langle s_{0, j}^{E}(\omega) s^{*}{ }_{k}^{E}(\omega)\right\rangle=$ $\left\langle s_{0, j}^{E}(\omega) I^{*}{ }_{j}(\omega)\right\rangle=\left\langle s_{j}^{E}(\omega) \xi^{*}{ }_{k}(\omega)\right\rangle+\left\langle\xi_{G}(\omega) \xi^{*}{ }_{k}(\omega)\right\rangle=0$ for $j \neq k$ and for $N \rightarrow \infty$ so as to neglect terms of order $1 / \mathrm{N}$ and higher, we obtain for the power spectrum of the spike train of the excitatory

$$
\begin{aligned}
& \left\langle s_{j}^{E}(\omega) s^{*_{j}^{E}}(\omega)\right\rangle=\left\langle s_{0, j}^{E}(\omega) s_{0, j}^{*^{E}}(\omega)\right\rangle+\sigma^{2}\left|A^{E}(\omega)\right|^{2} \\
& +\ldots+c \sigma^{2}\left|A^{E}(\omega)\right|^{2} \frac{2 \Re\left(\tilde{A}^{E} g e^{-i \omega \tau_{D}} \tilde{K}_{\tau}^{I} \tilde{A}^{I} \tilde{K}_{\tau}^{E}\right)-\left|\tilde{A}^{E} g \tilde{K}_{\tau}^{I} \tilde{A}^{I} \tilde{K}_{\tau}^{E}\right|^{2}}{\left|1-\tilde{A}^{E} g e^{-i \omega \tau_{D}} \tilde{K}_{\tau}^{I} \tilde{A}^{I} \tilde{K}_{\tau}^{E}\right|^{2}}
\end{aligned}
$$

\section{Acknowledgments}

We acknowledge support from the Lorentz Center (http://www. lc.leidenuniv.nl/) for organizing a workshop, which allowed us to discuss the results of this study with André Longtin and Nancy Kopell. We thank Magteld Zeitler for helpful discussions.

\section{References}

Ariaratnam, J. T., \& Strogatz, S. H. (2001). Phase diagram for the Winfree model of coupled nonlinear oscillators. Phys. Rev. Lett., 86, 4278-4281.

Baker, S. N., Pinches, E. M., \& Lemon, R. N. (2003). Synchronization in monkey motor cortex during a precision grip task. II: Effect of oscillatory activity on corticospinal output. J. Neurophysiol., 89, 1941-1953.

Börgers, C., \& Kopell, N. (2003). Synchronization in networks of excitatory and inhibitory neurons with sparse, random connectivity. Neural Comp., 15, 509-538.

Buzsáki, G., Leung, L. W. S., \& Vanderwolf, C. H. (1983). Cellular bases of hippocampal EEG in the behaving rat. Brain Res. Rev., 6, 139-171.

Doiron, B., Chacron, M. J., Maler, L., Longtin, A., \& Bastian J. (2003). Inhibitory feedback required for network oscillatory responses to communication but not prey stimuli. Nature, $421,539-543$.

Doiron, B., Lindner, B., Longtin, A., Maler, L., \& Bastian, J. (2004). Oscillatory activity in electrosensory neurons increases with the spatial correlation of the stochastic input stimulus. Phys. Rev. Lett., 93, 048101-048104.

Ermentrout, G. B., \& Kopell, N. (1986). Parabolic bursting in an excitable system coupled with a slow oscillation. SIAM J. Appl. Math., 46, 233-253.

Ernst, U., Pawelzik, K., \& Geisel, T. (1995). Synchronization induced by temporal delays in pulse-coupled oscillators. Phys. Rev. Lett., 74, 1570-1573.

Fries, P. (2005). A mechanism for cognitive dynamics: Neuronal communication through neuronal coherence. Trends in Cognitive Sciences, 9, 474-480.

Fries, P., Reynolds, J. H., Rorie, A. E., \& Desimone, R. (2001). Modulation of oscillatory neuronal synchronization by selective visual attention. Science, 291, 1560-1563.

Gray, C. M. (1994). Synchronous oscillations in neuronal systems: Mechanisms and functions. J. Comp. Neurosci., 1, 11-38. 
Gutkin, B. S., \& Ermentrout, G. B. (1998). Dynamics of membrane excitability determine interspike interval variability: A link between spike generation mechanisms and cortical spike train statistics. Neural Comp., 10, 1047-1065.

Hansel, D., \& Mato, G. (1993). Patterns of synchrony in a heterogeneous HodgkinHuxley neural-network with weak-coupling. Physica A, 200, 662-669.

Hansel, D., Mato, G., \& Meunier, C. (1995). Synchrony in excitatory neural networks. Neural Comp., 7, 307-337.

Hoppensteadt, F. C., \& Izhikevich, E. M. (1997). Weakly connected neural networks. New York: Springer-Verlag.

Lindner, B., Chacron, M. J., \& Longtin, A. (2005). Integrate-and-fire neurons with threshold noise: A tractable model of how interspike interval correlations affect neuronal signal transmission. Phys. Rev., E, 72, 021911.

Lindner, B., Doiron, B., \& Longtin, A. (2005). Theory of oscillatory firing induced by spatially correlated noise and delayed inhibitory feedback. Phys. Rev. E, 72, 061919.

Lindner, B., \& Schimansky-Geier, L. (2001). Transmission of noise coded versus additive signals through a neuronal ensemble. Phys. Rev. Lett., 86, 2934-2937.

Lindner, B., Schimansky-Geier, L., \& Longtin, A. (2002). Maximizing spike train coherence or incoherence in the leaky integrate-and-fire model. Phys. Rev. E, 66, 031916.

Lytton, W. W., \& Sejnowski, T. J. (1991). Simulations of cortical pyramidal neurons synchronized by inhibitory interneurons. J. Neurophysiol., 66, 1059-1079.

Mirollo, R. E., \& Strogatz, S. H. (1990). Synchronization of pulse-coupled biological oscillators. SIAM J. Appl. Math., 50, 1645-1662.

Natschlager, T., Maass, W., \& Zador, A. (2001). Efficient temporal processing with biologically realistic dynamic synapses. Network Computation in Neural Systems, $12,75-87$.

Pantic, L., Torres, J. J., \& Kappen, H. J. (2003). Coincidence detection with dynamic synapses. Network Computation in Neural Systems, 14(1), 17-33.

Reynolds, J. H., Chelazzi, L., \& Desimone, R. (1999). Competitive mechanisms subserve attention in macaque areas V2 and V4. J. Neurosci., 19, 1736-1753.

Reynolds, J. H., \& Desimone, R. (1999). The role of neural mechanisms of attention in solving the binding problem. Neuron, 24, 19-29.

Roelfsema, P., Lamme, V. A. F., \& Spekreijse, H. (2004). Synchrony and covariation of firing rates in the primary visual cortex during contour grouping. Nature Neurosci, 7, 982-991.

Rudolph, M., \& Destexhe, A. (2003). Tuning neocortical pyramidal neurons between integrators and coincidence detectors. J. Comp. Neurosci., 14, 239-251.

Schoffelen, J. M., Oostenveld, R., \& Fries, P. (2005). Neuronal coherence as a mechanism of effective corticospinal interaction. Science, 308, 111-113.

Senn, W., Segev, I., \& Tsodyks, M. (1998). Reading neuronal synchrony with depressing synapses. Neural Comp., 10, 815-819.

Singer, W. (1999). Neuronal synchrony: A versatile code for the definition of relations? Neuron, 24(1), 49-65.

Singer, W., \& Gray, C. M. (1995). Visual feature integration and the temporal correlation hypothesis. Annu. Rev. Neurosci., 18, 555-586. 
Traub, R. D., Whittington, M. A., Colling, S. B., Buzsaki, G., \& Jefferys, J. G. R. (1996). Analysis of gamma rhythms in the rat hippocampus in vitro and in vivo. J. Physiol. (London), 493, 471-484.

Tsodyks, M. V., \& Markram, H. (1997). The neural code between neocortical pyramidal neurons depends on neurotransmitter release probability. Proc. Natl. Ac. Sci. USA, 94, 719-723.

Tsodyks, M., Mitkov, I., \& Sompolinsky, H. (1993). Pattern of synchrony in inhomogeneous networks of oscillators with pulse interactions. Phys. Rev. Lett., 71, 1280-1283.

Tsodyks, M., Pawelzik, K., \& Markram, H. (1998). Neural networks with dynamic synapses. Neural Comp., 10, 821-835.

Tsodyks, M., Uziel, A., \& Markram, H. (2000). Synchrony generation in recurrent networks with frequency-dependent synapses. J. Neurosci., 20(RC50), 1-5.

van Vreeswijk, C. (2000). Analysis of the asynchronous state in networks of strongly coupled oscillators. Phys. Rev. Lett., 84, 5110-5113.

van Vreeswijk, C., \& Hansel, D. (2001). Patterns of synchrony in neural networks with spike adaptation. Neural Comp., 13, 959-992.

Whittington, M. A., Traub, R. D., \& Jeffreys, J. G. R. (1995). Synchronized oscillations in interneuron networks driven by metabotropic glutamate receptor activation. Nature, 373, 612-615.

Received January 30, 2006; accepted November 8, 2006. 